\title{
Digital breast tomosynthesis (DBT): recommendations from the Italian College of Breast Radiologists (ICBR) by the Italian Society of Medical Radiology (SIRM) and the Italian Group for Mammography Screening (GISMa)
}

\author{
Daniela Bernardi ${ }^{1} \cdot$ Paolo Belli $^{2} \cdot$ Eva Benelli $^{3} \cdot$ Beniamino Brancato $^{4} \cdot$ Lauro Bucchi $^{5} \cdot$ Massimo Calabrese $^{6} \cdot$

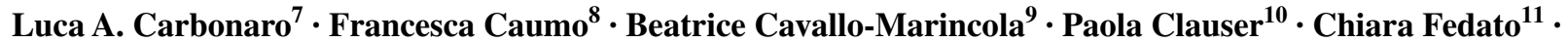 \\ Alfonso Frigerio ${ }^{12}$ - Vania Galli ${ }^{13}$ - Livia Giordano ${ }^{14}$ - Paolo Giorgi Rossi ${ }^{15}$ - Paola Golinelli ${ }^{16}$ - Doralba Morrone . $^{4}$ \\ Giovanna Mariscotti ${ }^{17} \cdot$ Laura Martincich $^{18} \cdot$ Stefania Montemezzi $^{19} \cdot$ Carlo Naldoni $^{20} \cdot$ Adriana Paduos $^{14}$. \\ Pietro Panizza $^{21}$ - Federica Pediconi ${ }^{9}$ - Fiammetta Querci ${ }^{22}$ - Antonio Rizzo ${ }^{23}$ - Gianni Saguatti ${ }^{24}$. \\ Alberto Tagliafico $^{25} \cdot$ Rubina M. Trimboli $^{26} \cdot$ Marco Zappa $^{27} \cdot$ Chiara Zuiani $^{28} \cdot$ Francesco Sardanelli $^{7,29}$ (i) \\ Received: 24 February 2017 / Accepted: 12 April 2017 / Published online: 25 May 2017 \\ (C) The Author(s) 2017. This article is an open access publication
}

\begin{abstract}
This position paper, issued by ICBR/SIRM and GISMa, summarizes the evidence on DBT and provides recommendations for its use. In the screening setting, DBT in adjunct to digital mammography (DM) increased detection rate by $0.5-2.7 \%$ and decreased false positives by $0.8-3.6 \%$ compared to DM alone in observational and double-testing experimental studies. The reduction in recall rate could be less prominent in those screening programs which already have low recall rates with DM. The increase in radiation exposure associated with DM/
\end{abstract}

Francesco Sardanelli

francesco.sardanelli@unimi.it

1 U.O. Senologia Clinica e Screening Mammografico, Dipartimento di Radiologia, APSS, Centro per i Servizi Sanitari, Pal. C, viale Verona, 38123 Trento, Italy

2 Dipartimento di Scienze Radiologiche, Università Cattolica del Sacro Cuore, Largo Agostino Gemelli 8, 00168 Rome, Italy

3 Zadig Scientific Communication Agency, Via Arezzo 21, 00161 Rome, Italy

4 Struttura Complessa di Senologia Clinica, Istituto per lo Studio e la Prevenzione Oncologica (ISPO), Via Cosimo il Vecchio 2, 50139 Florence, Italy

5 Romagna Cancer Registry, Romagna Cancer Institute (IRST) IRCCS, Via Piero Maroncelli 40, Meldola, 47014 Forlì, Italy

6 UOC Senologia Diagnostica, IRCCS AOU San Martino-IST, Largo Rosanna Benzi 10, 16132 Genoa, Italy

7 Unit of Radiology, IRCCS Policlinico San Donato, Via Morandi 30, San Donato Milanese, 20097 Milan, Italy

8 UOSD Breast Unit ULSS 20, Piazza Lambranzi 1, 37142 Verona, Italy
DBT protocols has been solved by the introduction of synthetic mammograms (sDM) reconstructed from DBT datasets. Thus, whenever possible, sDM/DBT should be preferred to DM/DBT. However, before introducing DBT as a routine screening tool for average-risk women, we should wait for the results of randomized controlled trials and for a statistically significant and clinically relevant reduction in the interval cancer rate, hopefully associated with a reduction in the advanced cancer rate. Otherwise, a potential for overdiagnosis and overtreatment cannot be

9 Dipartimento di Scienze Radiologiche, Oncologiche ed Anatomo-patologiche, Policlinico Umberto I, Sapienza Università di Roma, Viale Regina Elena 324, 00161 Rome, Italy

10 Division of Molecular and Gender Imaging, Department of Biomedical Imaging and Image-guided Therapy, Medical University of Vienna/General Hospital Vienna, Waehringer Guertel 18-20, 1090 Vienna, Austria

11 Regional Screening Coordinating Centre, Veneto Region, Venice, Italy

12 Regional Reference Centre for Breast Cancer Screening, Turin, Italy

13 Mammography Screening Centre, Local Health Authority, Modena, Italy

14 Epidemiology Unit, Centre for Cancer Prevention, Turin, Italy

15 Interinstitutional Epidemiology Unit, AUSL Reggio Emilia, and Arcispedale S. Maria Nuova, Reggio Emilia, Italy

16 Medical Physics Service, Local Health Authority, Modena, Italy 
excluded. Studies exploring this issue are ongoing. Screening of women at intermediate risk should follow the same recommendations, with particular protocols for women with previous BC history. In high-risk women, if mammography is performed as an adjunct to MRI or in the case of MRI contraindications, sDM/DBT protocols are suggested. Evidence exists in favor of DBT usage in women with clinical symptoms/signs and asymptomatic women with screen-detected findings recalled for work-up. The possibility to perform needle biopsy or localization under DBT guidance should be offered when DBT-only findings need characterization or surgery.

Keywords Breast cancer - Digital breast tomosynthesis . Mammography $\cdot$ Screening

\section{Introduction}

In the last years, many reports have appeared about digital breast tomosynthesis (DBT) in both the diagnostic and screening setting. In fact, thanks to a pseudo-threedimensional reconstruction, DBT allows for overcoming some limitations of standard two-dimensional (2D) digital mammography (DM) caused by structural overlapping and resulting into false-negative and false-positive findings [1]. The X-ray tube moves along an angular direction and generates multiple low-dose variably angled projections of the compressed breast. The reconstruction of multiple images of thin slices of the compressed breast is obtained by specialized software $[2,3]$. The technical solutions proposed by vendors differ for tube movement (continuous/discontinuous), width of oscillation angle, number of projections, type of detector, and other characteristics, although no substantial effects on diagnostic

17 Radiologia 1U, Dipartimento di Diagnostica per Immagini, Università di Torino, A. O. U. Città della Salute e della Scienza di Torino, Via Genova 3, 10126 Turin, Italy

18 U.O. Radiodiagnostica, Candiolo Cancer Institute, FPO, IRCCS, Strada Provinciale 142, km 3.95, Candiolo, 10060 Turin, Italy

19 DAI Patologia e Diagnostica, Azienda Ospedaliera Universitaria Integrata, Piazzale A. Stefani 1, 37126 Verona, Italy

20 Department of Health, Emilia-Romagna Region, Bologna, Italy

21 U.O. Radiologia Senologica, IRCCS Ospedale San Raffaele, Via Olgettina 60, 20132 Milan, Italy

22 Department of Prevention, Screening Centre, Local Health Authority, Sassari, Italy

23 Pathology Department, Local Health Authority, Asolo, Treviso, Italy performance related to these differences have been reported so far.

This position paper, issued by the Italian College of Breast Radiologists by the Italian Society of Medical Radiology (SIRM) and the Italian Group for Mammography Screening (GISMa), summarizes the available evidence on DBT and provides practical recommendations for its use. For the levels of evidence (LoE) reported here, we refer to the definitions given by the Centre for Evidence-Based Medicine, Oxford, United Kingdom [4].

\section{DBT for first-level screening}

Several studies have evaluated the potential of DBT in first-level screening [5-14]. In particular, three European prospective trials conducted in the context of organized population-based screening programs [6-9] and five retrospective studies from spontaneous screening settings [10-14] have shown that DBT combined with DM (or as a stand-alone approach [9]) allows for a better diagnostic performance than DM alone.

Results of DBT differ according to the study design, the screening interval, and the type of screening setting (organized versus spontaneous). A review [15] reported that DBT provides an increase in cancer detection rate from 0.5 to 2.7 per thousand screened women and a reduction in false-positive recall rate from 0.8 to 3.6 per 100 screened women. The possibility of using only the mediolateral oblique DBT projection, supported by one study [9], has the rationale of dose reduction. However, although many experiences have shown the superior diagnostic performance of two-view DBT compared with one-view DBT [16-18], the availability of synthetic 2D mammograms generated from the DBT dataset (see below) has substantially overcome the problem

24 Senology Unit, Local Health Authority, Bologna, Italy

25 Department of Experimental Medicine, DIMES, Institute of Anatomy, University of Genova, Via de Toni 14, 16132 Genoa, Italy

26 Integrative Biomedical Research PhD Program, Department of Biomedical Science for Health, Università degli Studi di Milano, Via Mangiagalli 31, 20133 Milan, Italy

27 UOC Epidemiologia Clinica, Istituto per lo Studio e la Prevenzione Oncologica (ISPO), Florence, Italy

28 Institute of Radiology, University of Udine, Piazzale S. M. della Misericordia 15, 33100 Udine, Italy

29 Department of Biomedical Sciences for Health, Università degli Studi di Milano, Via Morandi 30, San Donato Milanese, 20097 Milan, Italy 
of dose. A reduction in recall rate of greatly varying magnitude (from 6 to $82 \%$, median 31\%) has been found [19]. Notably, this variability depends on the baseline recall rate with standard DM because the higher the baseline DM recall rate, the higher the absolute and relative reduction obtained with DBT. Thus, the advantage of reducing the recall rate could be less prominent in those screening programs which already have low recall rates with standard DM.

If both DM and DBT are acquired (separately or with the so-called "combo" modality), the average glandular dose is approximately doubled. In fact, the DBT dose is similar to that of DM [20-22]. However, considering the dose reduction obtained through the introduction of DM as an alternative to film-screen mammography, the dose of these DM/DBT protocols too remains below the upper limit defined by the "European guidelines for quality assurance in breast cancer screening and diagnosis" published in 2006 [23].

Of note, radiation exposure would be an issue to take into consideration for a generalized adoption of DM/DBT protocols for population-based mass screening. The solution has come from the synthetic digital mammography (sDM) obtained through specialized algorithms summing and filtering the DBT datasets. Principles and methods have similarities with those generating maximum intensity projections in computed tomography and magnetic resonance imaging (MRI). These sDMs are virtual 2D mammograms obtained from DBT, paying no tradeoff in terms of radiation exposure [24-26].

Several authors have compared sDM/DBT protocols with DM/DBT protocols demonstrating a similar diagnostic performance [25-28]. Therefore, unless additional radiation exposure is specifically justified (as in the work-up of suspicious findings detected at DM), a diagnostic mammography examination can be performed using the sDM/ DBT approach.

Notably, two studies $[29,30]$ have reported that $54-57 \%$ of additional cancers detected by additional ultrasonography screening after negative DM were detected by DBT. This is a relevant argument in favor of DBT, considering the practical hurdles for a generalized mass screening with ultrasonography in adjunct to DM.

However, in the context of organized population-based screening programs, a simple increase in sensitivity and overall diagnostic performance of a new tool, even if statistically significant and clinically relevant, is not enough, per se, for its generalized adoption. According to the European Council Recommendation on cancer screening [31], evidence from randomized controlled trials is needed before introducing new screening tools. In particular, considering the pre-existing evidence in favor of screening mammography (recently confirmed by the International Agency for Research on Cancer [32]), caution is urged due to the possibility that a substantial part of the additional cancers detected by DBT could be overdiagnosed lesions, i.e. indolent malignant lesions that would never surface clinically during the woman's life [33]. Importantly, the consequence would be an increase in overtreatment, i.e. unnecessary surgery, radiation, and/or medical therapy.

It is worth to note that most of the additional cancers detected with DBT have been reported to be invasive [614]. A large study from the United States [10] has compared DM/DBT in approximately 174,000 women with DM alone in approximately 281,000 women. The DM/DBT approach was associated with a $29 \%$ increase in detection rate and a $15 \%$ reduction in recall rate. All the increase in cancer detection was due to invasive cancers $(+41 \%)$. No increase in the detection of ductal carcinoma in situ, deemed more probably overdiagnosed, was observed. However, we should consider that also invasive cancers can be indolent and overdiagnosed. Thus, no definitive conclusions can be drawn.

Several other issues cannot be ignored when discussing the potential introduction of DBT for screening. They include at least sufficient availability of DBT equipments, management of suspicious findings at DBT alone, and increased reading time [34-36], which implies the need for more breast radiologists involved as screening readers.

Thus, before introducing DBT as a first-level screening tool, we should wait for the results of randomized controlled trials (some of which are already ongoing in Italy) comparing a population sample having DM/DBT or sDM/ DBT at the first round and DM alone at the subsequent round with a population sample having DM alone at both rounds [37]. This will enable us to determine:

1. whether the expected increase in cancer detection at first round with DBT is associated or not associated with a reduction in interval cancer incidence;

2. whether the total incidence of advanced cancers (stage $\geq \mathrm{T} 2$ ) in the whole study period (i.e. considering all cancers detected in the first or second round as well as interval cancers) is lower in the DBT group compared with the control group; and

3. whether the total incidence of breast cancer in the DBT group exceeds that of the control group.

The attention paid to interval cancer rate is explained by the possibility to use this measure as a proxy of effectiveness in mortality reduction [15]. To our knowledge, only two articles $[38,39]$ have reported results concerning the interval cancer rate of DBT screening. The study design was a single-center retrospective analysis [38] and a multicenter [39] prospective cohort study. Comparing DM alone with DM/DBT screening, a reduction in interval cancer rate was observed in both studies, from 0.7 to 
0.5 per thousand screened women [38] and from 0.6 to 0.46 per thousand screened women [39], respectively. These variations were not statistically significant. However, it should be noted that neither study was designed and powered for interval cancer analysis, and that both were conducted in a spontaneous screening setting. These heavy limitations prevent us to draw any conclusion to be extrapolated to European organized biennial screening programs.

\section{DBT for work-up of screen-detected suspicious findings, as a first-line diagnostic examination in symptomatic women, for preoperative staging, and targeted evaluation after MRI}

Several studies [40-48] have shown that DBT is at least equivalent to additional DM views (magnification, spot compression, $90^{\circ}$ views, etc.), also reducing radiation exposure in both the diagnostic and screening setting. In symptomatic women, diagnostic accuracy is improved by DBT, reducing the number of suspicious findings and of unnecessary biopsies $[43,45,46]$. In the presence of palpable lesions, DBT has been reported to be never worse, and often better, than DM for estimating tumor size [49]. The better diagnostic performance of DBT is confirmed also in the case of invasive lobular cancers, as shown by a recent study [50]. In the preoperative setting, the combination of DM, DBT, and ultrasonography could provide the same information as MRI [51]. Finally, DBT allows for identifying some findings additionally found on preoperative MRI also when they are not visible at targeted ultrasonography, permitting a reduction in the number of MR-guided biopsies [52].

Studies evaluating inter-reader variability for DM/DBT versus DM alone have reported for each reader an increase in the detection rate and a reduction in the recall rate with an overall increase in the diagnostic performance [53-56]. In the particular context of spontaneous screening, DBT has also been found to reduce the number of short-time repeat examinations [55].

All these results allow for recommending DBT for symptomatic women and for work-up of screen-detected suspicious findings.

\section{Recommendations}

Recommendations are presented here for five categories of women:

1. asymptomatic women at average risk (first level of organized or spontaneous screening);
2. asymptomatic women at intermediate risk, including women with a previous breast cancer;

3. symptomatic women and women needing work-up of screen-detected suspicious findings;

4. asymptomatic women at hereditary/familial high risk;

5. asymptomatic women at high risk due to previous chest radiotherapy.

A sixth, final recommendation concerns needle biopsy under DBT guidance.

1. Asymptomatic women at average risk (first level of organized or spontaneous screening)

These women should undergo DM starting not before the age of 40 , with a screening interval that may vary according to local health authority's decision. As recently stated by the European Society of Breast Imaging [57, 58], direct DM should be preferred to film-screen mammography due to a reduced radiation exposure [59] and an at least equivalent diagnostic performance [60] (LoE A). The preference is also in favor of DM when compared with indirect digital phosphor storage plate (socalled computer radiography) [61].

DBT can be used as a first-level screening tool in women at average risk:

a) in the context of studies approved by an Ethical Committee, with enrollment after informed consent signature by the woman (for randomized controlled trials, refer to the scheme proposed by the Osservatorio Nazionale Screening [37]);

b) in the well-defined context of centers being part of public population-based screening programs, with previous experience with ethically approved studies concerning at least feasibility of screening DBT, demonstrated through articles published in peerreviewed journals.

In both cases, the sDM/DBT approach should be preferred (Loe A). In both cases, centers are requested to provide data regarding the screening process, service, and impact, including the women's compliance. Considering the data management needs, the necessary interactions with information technology services, and the impact of DBT on the whole multidisciplinary team, studies should be performed under umbrella of breast units. Moreover, usual monitoring data should be collected, in particular absolute and/or proportional incidence of interval cancer and of screen-detected cancers of stage $\mathrm{T} 2$ or higher [37, $62,63]$. Attention should be paid to avoid that the implementation of DBT studies causes a reduction in screening coverage, compliance, or quality indicators. 
2. Asymptomatic women at intermediate risk, including those with a previous breast cancer

Up to now, no substantial evidence has been produced in favor or against the use of DBT for screening women at intermediate risk of breast cancer, i.e. those with a lifetime risk from 15 to $19 \%$, estimated using multifactorial models. All these women should undergo screening DM with the same protocols recommended for asymptomatic women at average risk.

For asymptomatic women with a previous history of breast cancer (included in this general category of women at intermediate risk), we refer to the recommendations provided by the GISMa and the Italian College of Breast Radiologists by SIRM [64]. The recent observation of a reduction in indeterminate findings in surveillance after breast cancer treatment [65] plays in favor of DBT usage (LoE D) in this setting, with a preference for sDM/DBT protocols.

DBT can be used as a screening tool in women at intermediate risk:

a) in the context of studies approved by an Ethical Committee, with enrollment after informed consent signature by the woman;

b) in the well-defined context of centers having previous experience with ethically approved studies concerning DBT, demonstrated through articles published in peerreviewed journals.

Usual performance indicators should be collected, in particular absolute and/or proportional incidence of interval cancer and of screen-detected cancers of stage $\mathrm{T} 2$ or higher [36, 61, 62].

3. Symptomatic women and women needing work-up of screen-detected suspicious findings

We include in this category both women with suspicious clinical symptoms or signs (asking for a mammogram usually ordered by a general practitioner or a specialist) and asymptomatic women with screen-detected suspicious findings recalled for work-up in the context of spontaneous or organized screening. Women with previous history of breast cancer, if having suspicious clinical symptoms/signs or after a suspicious finding on an imaging study, are also included in this category.

In these women, if an indication to mammography exists and DBT is available, DBT should be performed (LoE A), preferably with a sDM/DBT protocol (LoE B). If sDM mammograms are not available, DBT can be performed after DM ( $\operatorname{LoE} B)$.
4. Asymptomatic women at hereditary/familial high risk

Women at hereditary/familial high risk should be screened in the context of dedicated pathways [66-71]. Considering the higher radiosensitivity of their breast tissue and the high sensitivity of MRI for breast cancer, mammography can be avoided at least up to 35 years of age, in particular in BRCA1 mutation carriers. If mammography is performed as an adjunct to MRI or in the case of MRI contraindications, a sDM/DBT protocol is suggested (LoE D). In all cases of mammographic work-up, DBT should be preferred to additional DM projections (LoE D).

5. Asymptomatic women at high risk due to previous chest radiotherapy

Recommendations regarding breast cancer screening for these women (mainly lymphoma survivors) were recently provided by the Italian College of Breast Radiologists by SIRM [72]. Both DM and contrast-enhanced MRI should be performed annually due to the suboptimal sensitivity of each of the two techniques [73-76]. A sDM/DBT protocol could be preferred to DM alone (LoE D).

\section{Needle biopsy under DBT guidance}

DBT can show doubtful/suspicious findings without any clinical correlate and even undetectable on DM, sDM, or ultrasonography [5-8]. This may occur in both the screening and diagnostic settings. In those cases, when the DBT finding is neither detectable at targeted ultrasonography nor at DM review, a needle biopsy (and, when necessary, also presurgical localization) should be performed under DBT guidance. Importantly, DBT guidance offers important advantages in terms of shorter procedure duration and reduced radiation exposure [77, 78] (LoE B).

As a consequence, centers offering DBT should also offer DBT-guided interventions for DBT findings not otherwise identifiable. These interventions can be performed at the same center where DBT was done or at another center functionally connected with the first one. At present, in fact, few centers are equipped with the device for DBTguided interventions.

\section{Conclusions}

Evidence available for DBT allows to recommend its usage for all cases of symptomatic women and women needing work-up of screen-detected suspicious findings (considering both spontaneous and organized screening). In these settings, when available, sDM/DBT protocols should be preferred for symptomatic women. 
Breast cancer screening using the current mammography technology has been demonstrated to be effective in reducing mortality from the disease. From this perspective, completing the shift from analog film-screen mammography and computed radiography systems, that are still in use, to direct digital mammography systems ranks first in the order of priorities.

A generalized adoption of DBT as a first-level screening tool should wait for a specific evidence, in particular for a statistically significant and clinically relevant reduction in interval cancer rate (hopefully associated with a reduction in advanced cancer rates). When this evidence will be available, both asymptomatic women at average and intermediate risk (including those with a previous breast cancer history) will be allowed to be screened with DBT on a routine basis. Accurate data collection will be necessary for many years in order to assess the overall impact of DBT technology in terms of mortality reduction and other efficacy/effectiveness indicators. For high-risk women, when a mammogram is indicated, a sDM/DBT protocol should be preferred.

The already existing evidence, which has been built with a non-negligible contribution of Italian breast radiologists, plays in favor of DBT. A trend for making DBT the mammography of the next future can be outlined. However, even in the United States, where FDA approved the use of some DBT devices in some cases for screening, the use of DBT is still quite limited. A recent survey [79] among the members of the Society of Breast Imaging reported that of 670 responders, only 200 (30\%) use DBT although $62 \%$ of non-users have planned to equip themselves with DBT.

Studies and researches are still needed for a deeper evaluation of DBT, a relevant innovation in breast imaging. In particular, the risk of an increased overdiagnosis and also several organizational issues (including increased reading time) suggest to wait for a more conclusive evidence before adopting DBT in population-based screening, a position shared with other experts and medical or health authority bodies worldwide $[15,57,58,80,81]$.

\section{Compliance with ethical standards}

Conflict of interest The authors declare no funding and no conflict of interest for this article.

Ethical standards This article does not contain any studies with human participants or animals performed by any of the authors.

Open Access This article is distributed under the terms of the Creative Commons Attribution 4.0 International License (http://creativecommons.org/licenses/by/4.0/), which permits unrestricted use, distribution, and reproduction in any medium, provided you give appropriate credit to the original author(s) and the source, provide a link to the Creative Commons license, and indicate if changes were made.

\section{References}

1. Vedantham S, Karellas A, Vijayaraghavan GR, Kopans DB (2015) Digital breast tomosynthesis: state of the art. Radiology 277:663-684

2. Baldwin P (2009) Digital breast tomosynthesis. Radiol Technol 81:57M-74M

3. Baker JA, Lo JY (2011) Breast tomosynthesis: state-of-the-art and review of the literature. Acad Radiol 18:1298-1310

4. Centre for Evidence-Based Medicine, Oxford, UK. www.cebm. net. Accessed 1 June 2016

5. Houssami N, Skaane P (2013) Overview of the evidence on digital breast tomosynthesis in breast cancer detection. Breast 22:101-108

6. Skaane P, Bandos AI, Gullien R et al (2013) Comparison of digital mammography alone and digital mammography plus tomosynthesis in a population-based screening program. Radiology 267:47-56

7. Skaane P, Bandos AI, Gullien R et al (2013) Prospective trial comparing full-field digital mammography (FFDM) versus combined FFDM and tomosynthesis in a population-based screening programme using independent double reading with arbitration. Eur Radiol 23:2061-2071

8. Ciatto S, Houssami N, Bernardi D et al (2013) Integration of 3D digital mammography with tomosynthesis for population breastcancer screening (STORM): a prospective comparison study. Lancet Oncol 14:583-589

9. Lang K, Andersson I, Rosso A et al (2015) Performance of one-view breast tomosynthesis as a stand-alone breast cancer screening modality: results from the Malmö Breast Tomosynthesis Screening Trial, a population-based study. Eur Radiol 26:184-190

10. Friedewald SM, Rafferty EA, Rose SL et al (2014) Breast cancer screening using tomosynthesis in combination with digital mammography. JAMA 311:2499-2507

11. Haas BM, Kalra V, Geisel J et al (2013) Comparison of tomosynthesis plus digital mammography and digital mammography alone for breast cancer screening. Radiology 269:694-700

12. McCarthy AM, Kontos D, Synnestvedt M et al (2014) Screening outcomes following implementation of digital breast tomosynthesis in a general-population screening program. J Natl Cancer Inst 106(11). doi:10.1093/jnci/dju316

13. Greenberg JS, Javitt MC, Katzen J, Michael S, Holland AE (2014) Clinical performance metrics of 3D digital breast tomosynthesis compared with 2D digital mammography for breast cancer screening in community practice. AJR Am J Roentgenol 203:687-693

14. Durand MA, Haas BM, Yao X et al (2015) Early clinical experience with digital breast tomosynthesis for screening mammography. Radiology 274:85-92

15. Houssami N (2015) Digital breast tomosynthesis (3D-mammography) screening: data and implications for population screening. Expert Rev Med Devices 12:377-379

16. Svahn T, Andersson I, Chakraborty D et al (2010) The diagnostic accuracy of dual-view digital mammography, single-view breast tomosynthesis and a dual-view combination of breast tomosynthesis and digital mammography in a free-response observer performance study. Radiat Prot Dosim 139:113-117

17. Wallis MG, Moa E, Zanca F et al (2012) Two-view and singleview tomosynthesis versus full-field digital mammography: 
high resolution X-ray imaging observer study. Radiology 262:788-796

18. Rafferty EA, Park JM, Philpotts LE et al (2014) Diagnostic accuracy and recall rates for digital mammography and digital mammography combined with one-view and two-view tomosynthesis: results of an enriched reader study. AJR Am J Roentgenol 202:273-281

19. Carbonaro LA, Di Leo G, Clauser P et al (2016) Impact on the recall rate of digital breast tomosynthesis as an adjunct to digital mammography in the screening setting. A double reading experience and review of the literature. Eur J Radiol 85:808-814

20. Cavagnetto F, Taccini G, Rosasco R et al (2013) 'In vivo' average glandular dose evaluation: one-to-one comparison between digital breast tomosynthesis and full-field digital mammography. Radiat Prot Dosim 157:53-61

21. Svahn TM, Houssami N (2015) Digital breast tomosynthesis in one or in two views as a replacement or adjunct technique to full-field digital mammography. Radiat Prot Dosim 165:314-320

22. Svahn TM, Houssami N, Sechopoulos I, Mattsson S (2015) Review of radiation dose estimates in digital breast tomosynthesis relative to those in two-view full field digital mammography. Breast 24:93-99

23. Perry N, Broeders M, de Wolf C et al (2006) European guidelines for quality assurance in breast screening and diagnosis. Fourth Edition. www.euref.org/downloads?download=24: $\quad$ europeanguidelines-for-quality. Accessed 7 Jan 2017

24. Gur D, Zuley ML, Anello MI et al (2012) Dose reduction in digital breast tomosynthesis (TM) screening using synthetically reconstructed projection images: an observer performance study. Acad Radiol 19:166-171

25. Skaane P, Bandos AI, Eben EB et al (2014) Two-view digital breast tomosynthesis screening with synthetically reconstructed projections images: comparison with digital breast tomosynthesis with full-field digital mammographic images. Radiology 271:655-663

26. Bernardi D, Macaskill P, Pellegrini M (2016) Breast cancer screening with tomosynthesis (3D mammography) with acquired or synthetic 2D mammography compared with 2D mammography alone (STORM-2): a population-based prospective study. Lancet Oncol 17:1105-1113

27. Choi JS, Han BK, Ko EY et al (2016) Comparison between twodimensional synthetic mammography reconstructed from digital breast tomosynthesis and full-field digital mammography for the detection of T1 breast cancer. Eur Radiol 26:2538-2546

28. Zuckerman SP, Conant EF, Keller BM et al (2016) Implementation of synthesized two-dimensional mammography in a population-based digital breast tomosynthesis screening program. Radiology 281:730-736

29. Nam KJ, Han BK, Ko ES et al (2015) Comparison of full-field digital mammography and digital breast tomosynthesis in ultrasonography-detected breast cancers. Breast 24:649-655

30. Tagliafico AS, Calabrese M, Mariscotti G et al (2016) Adjunct screening with tomosynthesis or ultrasound in women with mammography-negative dense breasts: Interim report of a prospective comparative trial. J Clin Oncol 34:1882-1888. doi:10.1200/JCO.2015.63.4147

31. European Council Recommendation on cancer screening, December 2, 2003 (2003/878/EC). https://ec.europa.eu/jrc/sites/ jrcsh/files/2_December_2003\%20cancer\%20screening.pdf. Accessed 22 Feb 2017

32. Lauby-Secretan B, Scoccianti C, Loomis D, International Agency for Research on Cancer Handbook Working Group et al (2015) Breast-cancer screening-viewpoint of the IARC Working Group. N Engl J Med 372:2353-2358

33. Colin C, Devouassoux-Shisheboran M, Sardanelli F (2014) Is breast cancer overdiagnosis also nested in pathologic misclassification? Radiology 273:625-652
34. Bernardi D, Ciatto S, Pellegrini M et al (2012) Application of breast tomosynthesis in screening: incremental effect on mammography acquisition and reading time. $\mathrm{Br} \mathrm{J}$ Radiol 85:e1174-e1178

35. Dang PA, Freer PE, Humphrey KL, Halpern EF, Rafferty EA (2014) Addition of tomosynthesis to conventional digital mammography: effect on image interpretation time of screening examinations. Radiology 270:49-56

36. Bernardi D, Ciatto S, Pellegrini M et al (2012) Prospective study of breast tomosynthesis as a triage to assessment in screening. Breast Cancer Res Treat 133:267-271

37. Osservatorio Nazionale Screening. Trial tomosintesi. http:// www.osservatorionazionalescreening.it/sites/default/files/allegati/documento $\% 20$ trial $\% 20$ tomosintesi_con $\% 20$ controlli $\% 20$ qualit\%C3\%A0.pdf\#overlay-context=node/59. Accessed 19 July 2016

38. McDonald ES, Oustimov A, Weinstein SP et al (2016) Effectiveness of digital breast tomosynthesis compared with digital mammography: outcomes analysis from 3 years of breast cancer screening. JAMA Oncol 2:737-743

39. Conant EF, Beaber EF, Sprague BL et al (2016) Breast cancer screening using tomosynthesis in combination with digital mammography compared to digital mammography alone: a cohort study within the PROSPR consortium. Breast Cancer Res Treat 156:109-116

40. Rafferty EA, Park JM, Philpotts LE et al (2013) Assessing radiologist performance using combined digital mammography and breast tomosynthesis compared with digital mammography alone: results of a multicenter, multireader trial. Radiology 266:104-113

41. Gilbert F, Tucker L, Gillan M et al (2015) The TOMMY trial: a comparison of tomosynthesis with digital mammography in the UK NHS Breast Screening Programme-a multicentre retrospective reading study comparing the diagnostic performance of digital breast tomosynthesis and digital mammography with digital mammography alone. Health Technol Assess 19:1-136

42. Morel JC, Iqbal A, Wasan RK et al (2014) The accuracy of digital breast tomosynthesis compared with coned compression magnification mammography in the assessment of abnormalities found on mammography. Clin Radiol 69:1112-1116

43. Tagliafico A, Astengo D, Cavagnetto F et al (2012) One-to-one comparison between digital spot compression view and digital breast tomosynthesis. Eur Radiol 22:539-544

44. Alakhras M, Bourne R, Rickard M et al (2013) Digital tomosynthesis: a new future for breast imaging? Clin Radiol 68:e225-e236

45. Bansal GJ, Young P (2015) Digital breast tomosynthesis within a symptomatic "one-stop breast clinic" for characterization of subtle findings. Br J Radiol 88(1053):20140855

46. Fornick D, Zackrisson S, Ljungberg O et al (2010) Breast tomosynthesis: accuracy of tumor measurement compared with digital mammography and ultrasonography. Acta Radiol 51:240-247

47. Cornford EJ, Turnbull AE, James JJ et al (2016) Accuracy of GE digital breast tomosynthesis vs supplementary mammographic views for diagnosis of screen-detected soft-tissue breast lesions. Br J Radiol 89(1058):20150735

48. Whelehan P, Heywang-Köbrunner SH, Vinnicombe SJ et al (2017) Clinical performance of Siemens digital breast tomosynthesis versus standard supplementary mammography for the assessment of screen-detected soft-tissue abnormalities: a multireader study. Clin Radiol 72:95.e9-95.e15

49. Timberg P, Bath M, Andersson I et al (2010) In-plane visibility of lesions using breast tomosynthesis and digital mammography. Med Phys 37:5618-5626

50. Mariscotti G, Durando M, Houssami N et al (2016) Digital breast tomosynthesis as an adjunct to digital mammography for 
detecting and characterizing invasive lobular cancers: a multireader study. Clin Radiol 71:889-895

51. Mariscotti G, Houssami N, Durando M et al (2014) Accuracy of mammography, digital breast tomosynthesis, ultrasound and MR imaging in preoperative assessment of breast cancer. Anticancer Res 34:1219-1226

52. Clauser P, Carbonaro A, Pancot $M$ et al (2015) Additional findings at preoperative breast MRI: the value of second look digital breast tomosynthesis. Eur Radiol 25:2830-2839

53. Bernardi D, Caumo F, Macaskill P et al (2014) Effect of integrating 3D-mammography (digital breast tomosynthesis) with 2D-mammography on radiologists' true-positive and false-positive detection in a population breast screening trial. Eur J Cancer 50:1232-1238

54. Caumo F, Bernardi D, Ciatto S et al (2014) Incremental effect from integrating 3D-ammography (tomosynthesis) with 2D-mammography: increased breast cancer detection evident for screening centres in a population-based trial. Breast 23:76-80

55. Rose SL, Tidwell AL, Ice MF et al (2014) A reader study comparing prospective tomosynthesis interpretations with retrospective readings of the corresponding FFDM examinations. Acad Radiol 21:1204-1210

56. Hakim CM, Catullo VJ, Chough DM et al (2015) Effect of the availability of prior full-field digital mammography and digital breast tomosynthesis images on the interpretation of mammograms. Radiology 276:65-72

57. Sardanelli F, Aase HS, Álvarez M et al (2016) Position paper on screening for breast cancer by the European Society of Breast Imaging (EUSOBI) and 30 national breast radiology bodies from Austria, Belgium, Bosnia and Herzegovina, Bulgaria, Croatia, Czech Republic, Denmark, Estonia, Finland, France, Germany, Greece, Hungary, Iceland, Ireland, Italy, Israel, Lithuania, Moldova, The Netherlands, Norway, Poland, Portugal, Romania, Serbia, Slovakia, Spain, Sweden, Switzerland and Turkey. Eur Radiol. doi:10.1007/s00330-016-4612-z

58. Sardanelli F, Fallenberg EM, Clauser P, European Society of Breast Imaging (EUSOBI), with language review by Europa Donna-The European Breast Cancer Coalition et al (2017) Mammography: an update of the EUSOBI recommendations on information for women. Insights Imaging 8:11-18

59. Gennaro G, di Maggio C (2006) Dose comparison between screen/film and full-field digital mammography. Eur Radiol $16: 2559-2566$

60. Pisano ED, Gatsonis C, Hendrick E, Digital Mammographic Imaging Screening Trial (DMIST) Investigators Group et al (2006) Diagnostic performance of digital versus film mammography for breast-cancer screening. N Engl J Med 353:1773-1783

61. Chelliah KK, Tamanang S, Bt Elias LS, Ying KY (2013) A comparative study of computed radiography-based mammography using digital phosphor storage plate and full field digital mammography. Indian J Med Sci 67:2328

62. Distante V, Frigerio A, Naldoni C et al (2006) Gruppo Italiano per lo Screening Mammografico. Documento di consenso GISMA in merito all'estensione dello screening mammografico di popolazione alla fascia di età 40-49 e 70-74. http://win.gisma. it/atti/bolognaconsenso05/documento_consenso.pdf. Accessed 7 Jan 2017

63. Giordano L, Giorgi D, Frigerio A, Gruppo Italiano per lo Screening Mammografico et al (2006) Process indicators and standards for the evaluation of breast cancer screening programmes. Epidemiol Prev 30(2 Suppl 1):5-9, 11-47

64. Bucchi L, Belli P, Benelli E et al (2016) Recommendations for breast imaging follow-up of women with a previous history of breast cancer: position paper from the Italian Group for Mammography Screening (GISMa) and the Italian College of Breast Radiologists (ICBR) by SIRM. 49. Radiol Med 121:891-896
65. Sia J, Moodie K, Bressel M et al (2016) A prospective study comparing digital breast tomosynthesis with digital mammography in surveillance after breast cancer treatment. Eur J Cancer $61: 122-127$

66. Sardanelli F, Giuseppetti GM, Canavese G et al (2008) Indications for breast magnetic resonance imaging. Consensus document "Attualità in senologia", Florence 2007. Radiol Med 113:1085-1095

67. Sardanelli F, Boetes C, Borisch B et al (2010) Magnetic resonance imaging of the breast: recommendations from the EUSOMA working group. Eur J Cancer 46:1296-1316

68. Sardanelli F, Podo F, Santoro F et al (2011) High Breast Cancer Risk Italian 1 (HIBCRIT-1) Study. Multicenter surveillance of women at high genetic breast cancer risk using mammography, ultrasonography, and contrast-enhanced magnetic resonance imaging (the high breast cancer risk italian 1 study): final results. Invest Radiol 46:94-105

69. Santoro F, Podo F, Sardanelli F (2014) MRI screening of women with hereditary predisposition to breast cancer: diagnostic performance and survival analysis. Breast Cancer Res Treat 147:685-687

70. Podo F, Santoro F, Di Leo G et al (2016) Triple-negative versus non-triple-negative breast cancers in high-risk women: phenotype features and survival from the HIBCRIT-1 MRI-including screening study. Clin Cancer Res 22:895-904

71. Mann RM, Balleyguier C, Baltzer PA, European Society of Breast Imaging (EUSOBI), with language review by Europa DonnaThe European Breast Cancer Coalition et al (2015) Breast MRI: EUSOBI recommendations for women's information. Eur Radiol 25:3669-3678

72. Mariscotti G, Belli P, Bernardi D et al (2016) Mammography and MRI for screening women who underwent chest radiation therapy (lymphoma survivors): recommendations for surveillance from the Italian College of Breast Radiologists by SIRM. Radiol Med 121:834-837

73. Ng AK, Garber JE, Diller LR et al (2013) Prospective study of the efficacy of breast magnetic resonance imaging and mammographic screening in survivors of Hodgkin lymphoma. J Clin Oncol 31:2282-2288

74. Tieu MT, Cigsar C, Ahmed S et al (2014) Breast cancer detection among young survivors of pediatric Hodgkin lymphoma with screening magnetic resonance imaging. Cancer 120:2507-2513

75. Sung JS, Lee CH, Morris EA, Oeffinger KC, Dershaw DD (2011) Screening breast MR imaging in women with a history of chest irradiation. Radiology 259:65-71

76. Freitas V, Scaranelo A, Menezes R et al (2013) Added cancer yield of breast magnetic resonance imaging screening in women with a prior history of chest radiation therapy. Cancer 119:495-503

77. Viala J, Gignier P, Perret B et al (2013) Stereotactic vacuumassisted biopsies on a digital breast 3D-tomosynthesis system. Breast J 19:4-9

78. Schrading S, Distelmaier M, Dirrichs T et al (2015) Digital breast tomosynthesis-guided vacuum-assisted breast biopsy: initial experiences and comparison with prone stereotactic vacuum-assisted biopsy. Radiology 274:654-662

79. Hardesty LA, Kreidler SM, Glueck DH (2016) Digital breast tomosynthesis utilization in the United States: a survey of physician members of the society of breast imaging. J Am Coll Radiol 13:R67-R73

80. Gilbert FJ, Tucker L, Young KC (2016) Digital breast tomosynthesis (DBT): a review of the evidence for use as a screening tool. Clin Radiol 71:141-150

81. Melnikow J, Fenton JJ, Miglioretti D, Whitlock EP, Weyrich MS (2016) Screening for breast cancer with digital breast tomosynthesis [Internet]. Agency for Healthcare Research and Quality (US), Rockville 